\title{
Effects of Silicon Carbide (SiC) Power Devices on HEV PWM Inverter Losses*
}

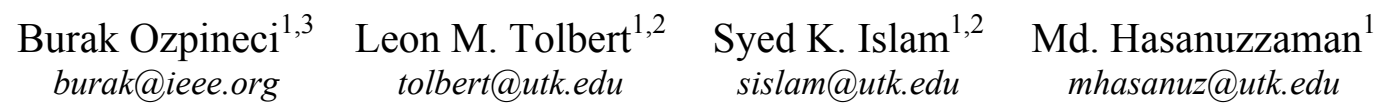 \\ ${ }^{1}$ Department of Electrical and \\ Computer Engineering \\ The University of Tennessee \\ ${ }^{2}$ Oak Ridge National Laboratory \\ P.O. Box 2009 \\ Oak Ridge, TN 37831-6472 \\ ${ }^{3}$ Oak Ridge Institute for Science \\ and Education \\ Oak Ridge, TN 37831-0117
}

Knoxville, TN 37996-2100

\begin{abstract}
The emergence of silicon carbide- (SiC-) based power semiconductor switches with their superior features compared with silicon $(\mathrm{Si})$ based switches has resulted in substantial improvements in the performance of power electronics converter systems. These systems with $\mathrm{SiC}$ power devices are more compact, lighter, and more efficient, so they are ideal for high-voltage power electronics applications, including hybrid electric vehicle (HEV) traction drives. In this paper, the effect of SiC-based power devices on HEV traction drive losses will be investigated. Reductions in heatsink size and device losses with the increase in the efficiency will be analyzed using an averaging model of a three-phase PWM inverter (TPPWMI). For more accurate results, device physics is taken into consideration to find the loss equations for the controllable switches.
\end{abstract}

\section{INTRODUCTION}

Presently, almost all the power electronics converter systems in automotive applications use silicon- ( $\mathrm{Si}-$ ) based power semiconductor switches. The performance of these systems is approaching the theoretical limits of the $\mathrm{Si}$ material. Another material, silicon carbide (SiC) with its superior properties compared with $\mathrm{Si}$, is a good candidate to be used in the next-generation power devices.

SiC-based power switches can be used in both electric traction drives and other automotive electrical subsystems with many benefits compared with Si based switches. With less than 1/100 the conduction drop, SiC-based devices have reduced conduction losses. Consequently, the efficiency of the power converter is higher. In addition, SiC-based semiconductor switches can operate at high temperatures (up to $600^{\circ} \mathrm{C}$ reported in [1]) without much change in their electrical properties. Thus the converter has a higher reliability. Reduced losses and allowable higher operating temperatures result in smaller heatsink size. Moreover, the high frequency operating capability of SiC converters lowers the filtering requirement and the filter size. As a result, they are compact, light, reliable, and efficient and have a high power density. These qualities satisfy the requirements of the automotive industry for power converters.

$\mathrm{SiC}$ comes in different crystalline structures or polytypes. The two most common polytypes are $6 \mathrm{H}-\mathrm{SiC}$ and $4 \mathrm{H}-\mathrm{SiC}$. First $6 \mathrm{H}-\mathrm{SiC}$ was available; then $4 \mathrm{H}-\mathrm{SiC}$ was introduced around 1994, and it deflected interest from $6 \mathrm{H}-$ $\mathrm{SiC}$ for high-power applications. In this paper, $\mathrm{SiC}$ refers to $4 \mathrm{H}-\mathrm{SiC}$

The number of $\mathrm{SiC}$ publications has been increasing rapidly in the last few years. There are many examples

*This research was supported in part by an appointment to the Oak Ridge National Laboratory Postmaster's Research Participation Program administered jointly by the Oak Ridge Institute for Science and Education and Oak Ridge National Laboratory. of $4 \mathrm{H}-\mathrm{SiC}$ and $6 \mathrm{H}-\mathrm{SiC} \mathrm{PiN}$ diodes, Schottky diodes, IGBTs, thyristors, BJTs, various MOSFETs, GTOs, MCTs, MTOs, etc. in $\mathrm{kV}$ range with reduced onresistances. However, except for some of the diodes, these are all experimental devices with very low current ratings. Few papers have been published on power converter applications of $\mathrm{SiC}$ diodes [2] and none on the applications of controlled switches. As of September 2001, two companies have advertised the commercial availability of $\mathrm{SiC}$ Schottky diodes, Infineon $(600 \mathrm{~V}$ up to $6 \mathrm{~A}$ or $300 \mathrm{~V}$ up to $10 \mathrm{~A})$ and Microsemi (100-200-480V, 1A). However, these are not for sale in the United States yet.

At Oak Ridge National Laboratory (ORNL), a SiC power MOSFET is currently being designed. This power device will be used in power electronics converter systems for automotive applications to demonstrate the benefits of $\mathrm{SiC}$-based power devices. One of the selected automotive applications for this project is a traction drive. In addition, system modeling of automotive power electronics systems using SiC-based devices instead of Si-based devices is being conducted. New gate drive layouts, circuit topologies, and filter requirements will also be developed to take advantage of the special properties of $\mathrm{SiC}$ devices.

\section{ADVANTAGES OF SiC COMPARED WITH Si}

As mentioned before, SiC power devices, with their close-to-ideal characteristics, bring great performance improvements. Some of these advantages compared with $\mathrm{Si}$ based power devices are as follows:

- SiC devices are thinner and they have lower onresistances. At low breakdown voltages $(\sim 50 \mathrm{~V}), \mathrm{SiC}$ unipolar device on-resistances are around 100 times less; and at higher breakdown voltages $(\sim 5000)$, up to 300 times less [3]. With lower $R_{o n}$, SiC power devices have lower conduction losses (Fig. 1) and therefore higher overall efficiency.

- SiC-based power devices have higher breakdown voltages because of their higher electric breakdown field.

- $\quad \mathrm{SiC}$ has a higher thermal conductivity and thus a lower junction-to-case thermal resistance, $R_{t h-j c}$ and thus device temperature increase is slower.

- $\mathrm{SiC}$ can operate at high temperatures. SiC device operation at up to $600^{\circ} \mathrm{C}$ is mentioned in literature [1]. $\mathrm{Si}$ devices, on the other hand, can operate at a maximum junction temperature of only $150^{\circ} \mathrm{C}$.

- $\mathrm{SiC}$ is extremely radiation hard; i.e., radiation does not degrade the electronic properties of SiC.

- Forward and reverse characteristics of $\mathrm{SiC}$ power devices vary only slightly with temperature and time; therefore, they are more reliable. 

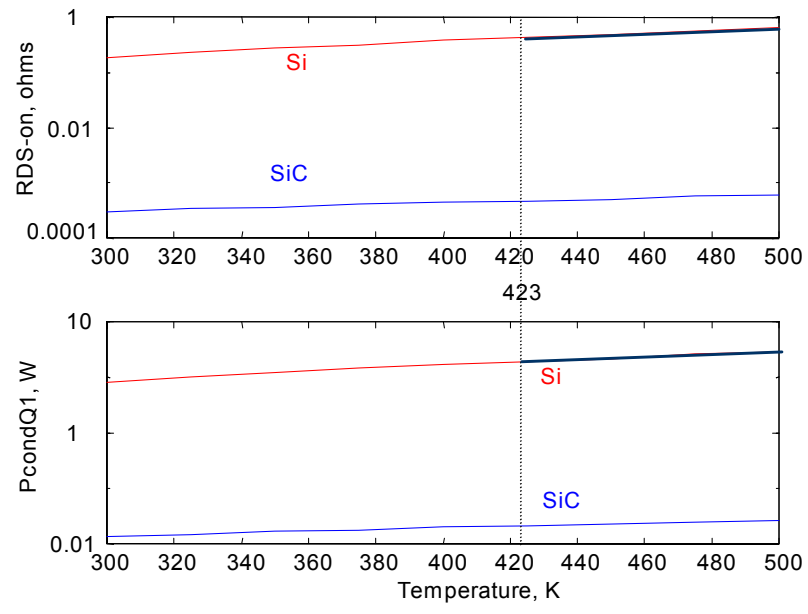

Fig. 1: The change in MOSFET on resistance and conduction losses with temperature. Note that $\mathrm{Si}$ cannot withstand temperatures of over $423^{\circ} \mathrm{K}$

$\left(150^{\circ} \mathrm{C}\right)(\mathrm{Si}-\mathrm{red}, 4 \mathrm{H}$-SiC-blue, logarithmic y-axis)

- $\quad$ SiC-based devices have excellent reverse recovery characteristics [4]. With less reverse recovery current, the switching losses and EMI are reduced and there is less or no need for snubbers. Typical turn-off waveforms of commercial $\mathrm{Si}$ and $\mathrm{SiC}$ diodes are given in Fig. 2.

\section{LOSS CALCULATIONS}

For the extensive study of the thermal advantages of using SiC-based power devices in HEV power electronics, a three-phase PWM inverter (TPPWMI) (Fig. 3) is selected. TPPWMI supplies power to the traction motors in an HEV. It consists of six MOSFETs and their six antiparallel diodes. To show the thermal advantages of using SiC-based power devices in HEVs, the losses on each device needs to be calculated over a practical drive schedule. The drive schedule generally used is the Federal Urban Driving Schedule (FUDS). The FUDS cycle is a 1370 -second velocity profile of an average person's vehicle on the way to work from home.

The calculation of device losses over the FUDS cycle is an involved and time-consuming procedure for a PWM inverter. This is because of the high sampling frequency required to simulate the high frequency switching PWM inverter. For a $20 \mathrm{kHz}$ switching frequency, the simulation sampling time would be on the order of $0.2 \times 10^{-6}$ seconds.

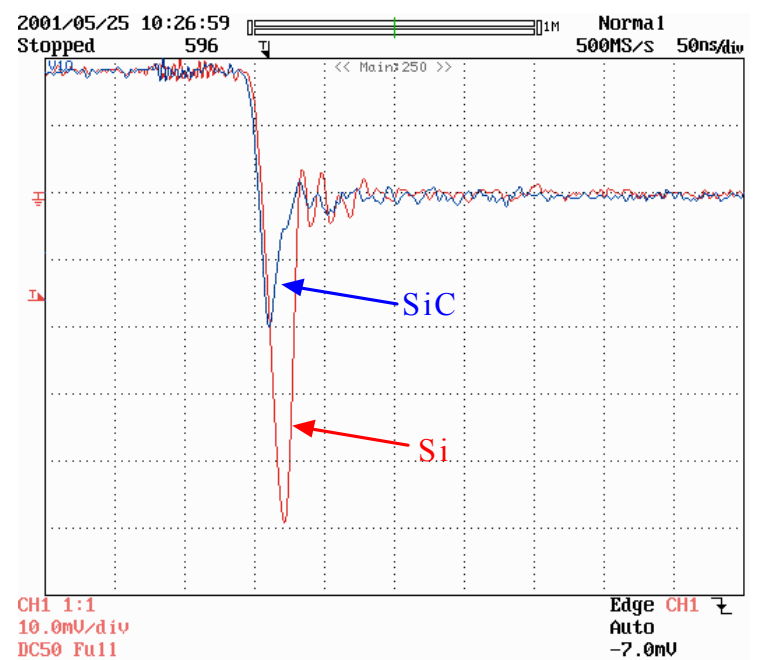

Fig. 2: Typical experimental Si and SiC diode reverse recovery

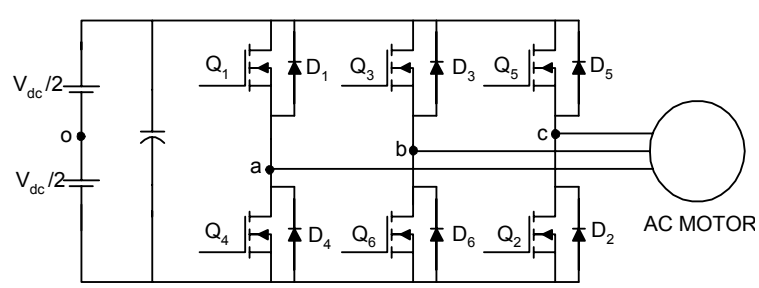

Fig. 3: Three-phase inverter driving and induction machine load.

Over the FUDS cycle, this means $6.9 \times 10^{9}$ points to iterate if a fixed sample rate is used. Thus, it would be impractical to do the loss calculations using commercial simulation packages like PSpice because the calculation would take a tremendous amount of time. In literature, an averaging technique that gives a good estimation of the behavior of the converter at a shorter time has been proposed $[5,6]$.

\section{A. Average Modeling of TPPWMI}

An output voltage waveform and its construction for a PWM inverter is given in Fig. 4 for a switching period of $T_{c}$. In this case, the modulating wave $v_{a o} *$ is assumed to be equal to a constant, $K$, during $T_{c}$ period. This assumption is valid when the output period, $T_{o}$ is more than ten times greater than the switching period, $T_{c}$. The averaging technique uses the same assumption. At every $T_{c}$, the variables are averaged; and the average value is assumed to be the constant value of the same variable over $T_{c}$.

Table I gives the meanings of the variables used in loss calculations.

Averaging $v_{a o}$ over $T_{c}$ gives

$\bar{v}_{a o}=\frac{1}{T_{c}} \int_{0}^{T_{c}} v_{a o} d t=K \frac{V_{d c}}{2}$

In practice, $v_{a o} *$ is a sinusoidally varying waveform with a peak value of $M$, where $M$ is the modulation index.

$v_{a o}=M \sin \theta$

Thus,

$\bar{v}_{a o}=M \sin \theta \frac{V_{d c}}{2}$

$\bar{v}_{b o}$ and $\bar{v}_{c o}$ can be found by delaying $\bar{v}_{a o}$ by $2 \pi / 3$ and $4 \pi / 3$ respectively.

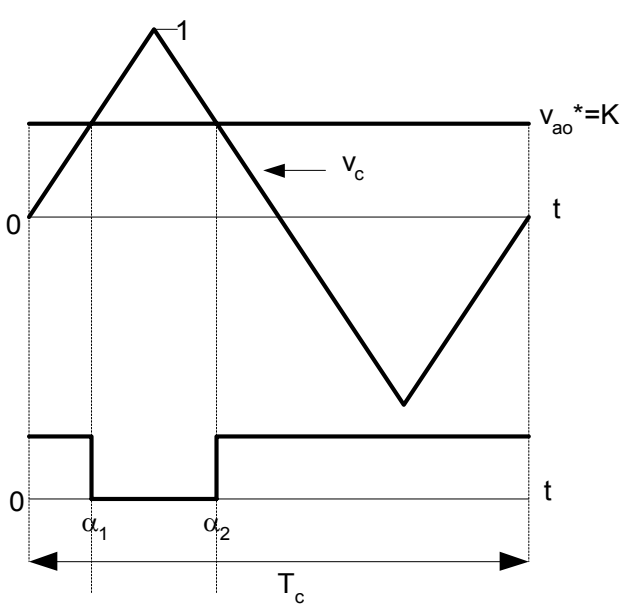

Fig. 4: PWM output voltage waveform 
$\bar{v}_{b o}=M \sin \left(\theta-\frac{2 \pi}{3}\right) \frac{V_{d c}}{2}, \bar{v}_{c o}=M \sin \left(\theta-\frac{4 \pi}{3}\right) \frac{V_{d c}}{2}$

Thus, $\bar{v}_{a o}$ is a stepped waveform sampled at a frequency of $f_{c}=1 / T_{c}$, and each step corresponds to the average of the actual $v_{a o}$ in the same interval, $T_{c}$. This means that instead of the chopped $v_{a o}$ waveform in Fig. 4, it can be assumed that $v_{a o}$ had a constant stepped value and this value is the average of $v_{a o}$, i.e. $\bar{v}_{a o}$. The same is also true for $\bar{v}_{b o}$ and $\bar{v}_{c o}$.

These averaged voltages are fed to a three-phase induction machine to validate the accuracy of the averaging technique. Fig. 5 shows the comparison of the average model with the actual model. The average model waveform tracks the actual waveform with great accuracy.

\section{B. Average loss modeling of TPPWMI}

The main losses on the power devices are conduction losses and switching losses. These losses will be calculated separately in the following subsections for diodes and MOSFETs in TPPWMI.

\section{1). MOSFET losses:}

$\underline{\text { Conduction losses: }}$

Conduction losses of a MOSFET $Q_{l}$ are given by

$P_{\text {cond, } Q 1}=I_{Q 1, r m s}^{2} \cdot R_{D S, o n}$

$I_{Q 1, r m s}^{2}$ can be found directly by,

$I_{Q 1, r m s}=\sqrt{\frac{1}{N} \sum_{n=0}^{N-1} i_{o, n}^{2} D_{n}}$

where $D_{n}=$ duty ratio in the nth interval

$i_{o, n}=$ average output current in the nth interval

TABLE I:

NOMENCLATURE

\begin{tabular}{|c|c|}
\hline$g_{m}$ & $=$ transconductance of the $\operatorname{MOSFET}\left(\Omega^{-1}\right)$ \\
\hline$B V$ & $=$ breakdown voltage $(\mathrm{V})$ \\
\hline$E_{c}$ & $=$ avalanche breakdown electric field $(\mathrm{V} / \mathrm{cm})$ \\
\hline$J^{\prime}$ & $=$ peak drain current density $\left(\mathrm{A} / \mathrm{cm}^{2}\right)$ \\
\hline$V$ & $=$ applied voltage $(\mathrm{V})$ \\
\hline$V_{G H}, V_{G L}$ & $\begin{aligned}= & \text { Highest and lowest applied gate voltages of } \\
& \text { the MOSFET }(\mathrm{V})\end{aligned}$ \\
\hline$V_{t h}$ & $=$ threshold voltage of the MOSFET $(\mathrm{V})$ \\
\hline$\varepsilon_{s}$ & $=$ permittivity of the semiconductor $(\mathrm{F} / \mathrm{cm})$ \\
\hline$R_{D s, o n}$ & $=$ on resistance of the MOSFET $(\Omega)$ \\
\hline$I$ & $=$ peak drain current $(\mathrm{A})$ \\
\hline$M$ & $=$ modulation index \\
\hline$\phi$ & $=$ current phase angle (radians) \\
\hline$I_{R}$ & $=$ peak reverse recovery current of the diode $(\mathrm{A})$ \\
\hline$V_{R}$ & $=$ reverse voltage applied to the diode $(\mathrm{V})$ \\
\hline$R_{D}$ & $=$ on resistance of the diode \\
\hline$V_{D}$ & $=$ voltage drop of the diode \\
\hline$t_{r r}$ & $=$ total reverse recovery time $(\mathrm{s})$ \\
\hline$t_{a}, t_{b}$ & $=$ defined in Fig. 6 \\
\hline$f_{c}$ & $=$ switching frequency $(\mathrm{Hz})$ \\
\hline$T_{c}$ & $=$ switching period $(\mathrm{s})$ \\
\hline$S$ & $=$ softness factor $t_{b} / t_{a}$ \\
\hline$A$ & $=$ chip area $\left(\mathrm{cm}^{2}\right)$ \\
\hline$f_{o}, T_{o}$ & $=$ output voltage frequency and period \\
\hline$f_{c}, T_{c}$ & $=$ switching frequency and period \\
\hline$T_{e}$ & $=$ torque developed by the electric machine \\
\hline$\omega_{r}$ & $=$ mechanical speed of the electric machine \\
\hline$\eta$ & $=$ efficiency of the electric machine \\
\hline$p$ & $=$ number of poles of the electric machine \\
\hline
\end{tabular}

$N=\frac{f_{c}}{f_{o}}=\frac{T_{o}}{T_{c}}$

$i_{o, n}=I \sin \left(\theta_{n}-\phi\right), I=$ peak output current

$\theta_{n}=\frac{2 \pi n}{N}, \phi=$ phase angle of the current

$D_{n}=\frac{1}{2}\left(1+M \sin \theta_{n}\right) \quad$ (see Fig. 4)

For $N \gg>1$, the summation in (5) can be approximated by an integral

$$
\begin{aligned}
I_{Q 1, r m s} & \cong I \sqrt{\frac{1}{2 \cdot 2 \pi} \int_{\phi}^{\pi+\phi} \sin ^{2}(\theta-\phi)(1+M \sin \theta) d \theta} \\
& =I \sqrt{\frac{1}{4 \pi}\left(\frac{\pi}{2}+\frac{4}{3} M \cos \phi\right)} \\
& =I \sqrt{\frac{1}{8}+\frac{1}{3 \pi} M \cos \phi}
\end{aligned}
$$

Thus,

$$
P_{\text {cond }, Q 1}=I^{2} \cdot R_{D S, \text { on }} \cdot\left(\frac{1}{8}+\frac{1}{3 \pi} M \cos \phi\right)
$$

Note that the integration limits are from $\phi$ to $\pi+\phi$ because, during that interval the current polarity is positive and $\mathrm{Q}_{1}$ conducts when turned on.

\section{Switching losses:}

Most switching loss calculations reported in literature use an approximate linear model for device turn-on and off. This does not consider the device physics. In this paper, on the other hand, physics-based energy loss equations from [7] will be considered to calculate the MOSFET switching losses. Energy loss during switching in a MOSFET is expressed as follows:
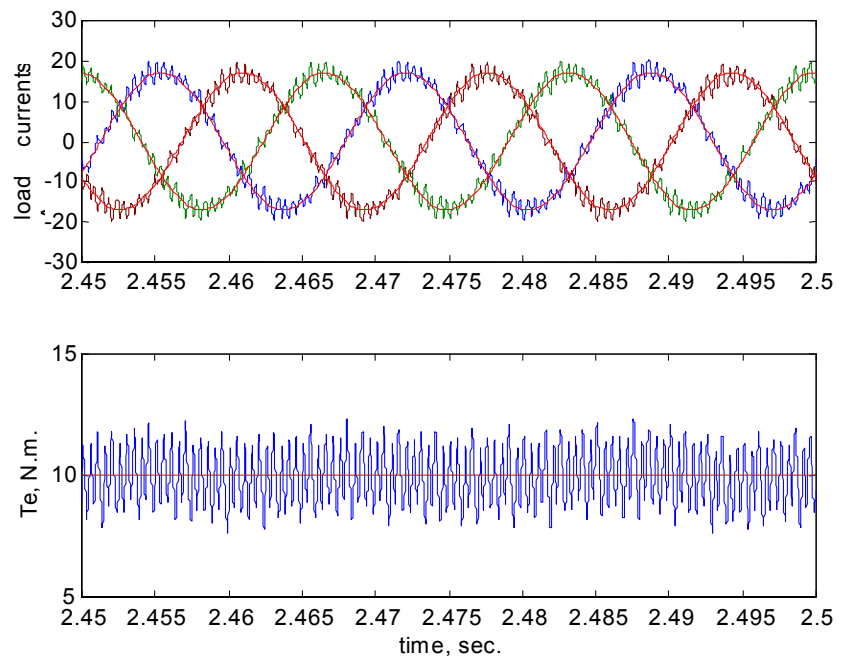

Fig. 5: Comparison of the average model waveforms with the actual ones 


$$
\begin{aligned}
E_{t o t} & =E_{\text {on }}+E_{\text {off }} \\
& =\frac{1}{3\left(K_{1}-1\right)} \varepsilon_{S} E_{C} V\left(\frac{V}{B V}\right)^{1 / 2}+\frac{1}{3\left(K_{2}+1\right)} \varepsilon_{S} E_{C} V\left(\frac{V}{B V}\right)^{1 / 2} \\
& =D\left(\frac{J}{C_{1}-J}+\frac{J}{C_{2}+J}\right)
\end{aligned}
$$

where $\quad K_{1}=\frac{g_{m}\left(V_{G H}-V_{t h}\right)}{J}, \quad K_{2}=\frac{g_{m}\left(V_{t h}-V_{G L}\right)}{J}$,

$D=\frac{1}{3} \varepsilon_{S} E_{C} V\left(\frac{V}{B V}\right)^{1 / 2}, C_{1}=g_{m}\left(V_{G H}-V_{t h}\right)$,

$C_{2}=g_{m}\left(V_{t h}-V_{G L}\right)$,

and $J=\frac{i_{O}}{A}=\frac{I}{A} \sin (\theta-\phi)=J^{\prime} \sin (\theta-\phi)$

$Q_{l}$ switching loss in one $T_{c}$ period is

$p_{Q 1}=\frac{E_{\text {on }}+E_{\text {off }}}{T_{\mathcal{C}}}=f_{\mathcal{C}} E_{t o t}$

Averaging over the output period, $T_{o}$,

$$
\begin{aligned}
P_{s w, Q 1} & =\frac{1}{N} \sum_{n=1}^{N} f_{C} E_{t o t}(n) \cong \frac{1}{2 \pi} \int_{\phi}^{\pi+\phi} f_{C} E_{t o t} d \theta \\
& \cong \frac{D f_{C}}{2 \pi}\left[\frac{C_{1}}{\sqrt{C_{1}^{2}-J^{\prime 2}}}\left(\pi+2 \tan ^{-1}\left(\frac{J^{\prime}}{\sqrt{C_{1}^{2}-J^{\prime 2}}}\right)\right)\right. \\
& +\frac{C_{2}}{\sqrt{C_{2}^{2}-J^{\prime 2}}}\left(-\pi+2 \tan ^{-1}\left(\frac{J^{\prime}}{\sqrt{C_{2}^{2}-J^{\prime 2}}}\right)\right)
\end{aligned}
$$

Note that all six MOSFETs have the same switching and conduction losses for a balanced three-phase load. To find the total MOSFET losses of the inverter, $P_{c o n d, Q I}$ and $P_{s w, Q I}$ should be added and the result should be multiplied by 6 .

\section{2) Diode losses}

Conduction losses:

Conduction losses of diode $D_{4}$ are given by

$P_{\text {cond }, D 4}=I_{D 4, a v} \cdot V_{D}+I_{D 4, r m s}^{2} \cdot R_{D}$

The expression to find $I_{D 4, \text { rms }}$ is the same as the expression to find $I_{Q 1, r m s}$ except for the duty ratio. $D_{4}$ conducts when the current is positive and $Q_{l}$ is off; therefore the duty ratio for $D_{4}$ is $1-D_{n}=\frac{1}{2}\left(1-M \sin \theta_{n}\right)$.

Then, $I_{D 4, r m s}=I \sqrt{\frac{1}{8}-\frac{1}{3 \pi} M \cos \phi}$

The average diode current can be found by averaging as follows:

$$
\begin{aligned}
I_{D 4, a v} & =\frac{1}{N} \sum_{n=0}^{N-1} i_{O, n}\left(1-D_{n}\right) \\
& \cong \frac{1}{2 \pi} \int_{\phi}^{\pi+\phi} I \sin (\theta-\phi) \frac{1}{2}(1-M \sin \theta) d \theta \\
& =I\left(\frac{1}{2 \pi}-\frac{M \cos \phi}{8}\right)
\end{aligned}
$$

Thus,

$$
P_{\text {cond }, D 4}=I^{2} \cdot R_{D} \cdot\left(\frac{1}{8}-\frac{1}{3 \pi} M \cos \phi\right)+I \cdot V_{D} \cdot\left(\frac{1}{2 \pi}-\frac{1}{8} M \cos \phi\right)(18)
$$

Switching losses:

The most important part of the diode switching losses is the reverse recovery losses. The rest of the losses are negligible. Reverse recovery losses will be calculated using the linearized turn-off waveforms in Fig. 6.

Average loss in a switching period, $T_{c}$, is

$$
\begin{aligned}
p_{D 4} & =\frac{1}{T_{c}} \int_{0}^{T_{C}} v_{s w} i_{s w} d t \\
& =\frac{1}{T_{C}} \int_{a}^{b}\left(-V_{R}\right) \cdot I_{R} \cdot\left(-1+\frac{t-a}{t_{b}}\right) d t=f_{c} \frac{V_{R} I_{R} t_{b}}{2}
\end{aligned}
$$

Find $t_{b}$ and $I_{R}$ in terms of $S$ and $t_{r r}$ $S \equiv \frac{t_{b}}{t_{a}} \quad$ and $\quad t_{b}+t_{a}=t_{r r}, \quad$ therefore $\quad t_{b}=\left(\frac{S}{S+1}\right) t_{r r}$ and $t_{a}=\left(\frac{1}{S+1}\right) t_{r r}$

$I_{R}$ can be calculated as follows,

$I_{R}=\frac{d I_{F}}{d t} t_{a}=\frac{d I_{F}}{d t} \frac{1}{S+1} t_{r r}$

Then,

$$
\begin{aligned}
p_{D 4} & =\frac{f_{C} V_{R}}{2}\left(\frac{d I_{F}}{d t}\right) \frac{1}{S+1} t_{r r} \frac{S}{S+1} t_{r r} \\
& =\frac{f_{c} V_{R}}{2 S}\left(\frac{d I_{F}}{d t}\right)\left(\frac{S t_{r r}}{S+1}\right)^{2}
\end{aligned}
$$

The variables $S$ and $t_{r r}$ in (22) are relatively independent of $I_{F}$, and $\left(d I_{F} / d t\right)$ does not depend on $I_{F}$, either. $\left(d I_{F} / d t\right)$ is circuit dependent: $\left(d I_{F} / d t\right)=E / L$. Thus, the average $D_{4}$ switching loss in an output period, $T_{o}$, is

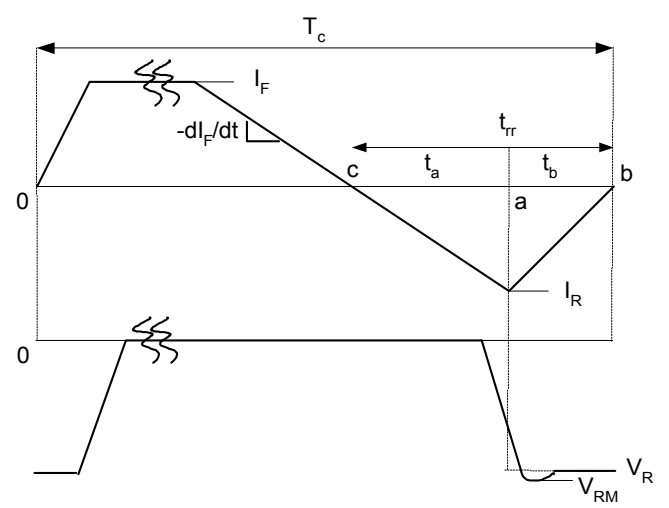

Fig 6: Typical diode turn-off waveform 


$$
\begin{aligned}
P_{s w, D 4} & =\frac{1}{N} \sum_{n=1}^{N} \frac{f_{\mathcal{C}} V_{R}}{2 S}\left(\frac{d I_{F}}{d t}\right)\left(\frac{S t_{r r}}{S+1}\right)^{2} \\
& =\frac{f_{c} V_{R}}{2 S}\left(\frac{d I_{F}}{d t}\right)\left(\frac{S t_{r r}}{S+1}\right)^{2}
\end{aligned}
$$

The diode reverse recovery current also contributes to the average $Q_{l}$ conduction losses. This contribution can be calculated by averaging the reverse recovery current in the switching period,

$$
\begin{aligned}
i_{D 4 \rightarrow Q 1, r m s} & =\sqrt{\frac{I_{R}^{2}}{T_{c}}\left(\int_{c}^{a}\left(\frac{c-t}{t_{a}}\right)^{2} d t+\int_{a}^{b}\left(1-\frac{t-a}{t_{b}}\right)^{2} d t\right)} \\
& =I_{R} \sqrt{\frac{t_{a}+t_{b}}{3 T_{c}}} \\
& =\left(\frac{d I_{F}}{d t}\right) \frac{t_{r r}}{S+1} \sqrt{\frac{t_{r r}}{3 T_{c}}}
\end{aligned}
$$

Thus, the conduction loss contributed to $Q_{1}$ by $D_{4}$ is

$$
\begin{aligned}
P_{\text {cond }, D 4 \rightarrow Q 1} & =i_{D 4-Q 1, r m s}^{2} R_{D S, \text { on }} \\
& =\frac{t_{r r}}{3 T_{c}}\left[\left(\frac{d I_{F}}{d t}\right) \frac{t_{r r}}{S+1}\right]^{2} R_{D S, o n}
\end{aligned}
$$

3) Device loss equations (summary): MOSFET losses:

$$
\begin{aligned}
P_{\text {tot }}^{M O S F E T} & =P_{\text {cond }, Q 1}+P_{\text {sw, } Q 1}+P_{\text {cond }, D 4 \rightarrow Q 1} \\
& =I^{2} \cdot R_{D S, \text { on }} \cdot\left(\frac{1}{8}+\frac{1}{3 \pi} M \cos \phi\right) \\
& +\frac{D f_{c}}{2 \pi}\left[\frac{C_{1}}{\sqrt{C_{1}^{2}-J^{\prime 2}}}\left(\pi+2 \tan ^{-1}\left(\frac{J^{\prime}}{\sqrt{C_{1}^{2}-J^{\prime 2}}}\right)\right)\right. \\
& +\frac{C_{2}}{\sqrt{C_{2}^{2}-J^{\prime 2}}}\left(-\pi+2 \tan ^{-1}\left(\frac{J^{\prime}}{\left.\left.\sqrt{C_{2}^{2}-J^{\prime 2}}\right)\right)}\right)\right] \\
& +\frac{t_{b}}{3 T_{c}}\left[\left(\frac{d I_{F}}{d t}\right) \frac{t_{r r}}{S+1}\right]^{2} R_{D S, \text { on }}
\end{aligned}
$$

Diode losses:

$$
\begin{aligned}
P_{\text {tot }}^{D I O D E} & =P_{\text {cond }, D 4}+P_{s w, D 4} \\
& =I^{2} \cdot R_{D} \cdot\left(\frac{1}{8}-\frac{1}{3 \pi} M \cos \phi\right)+I \cdot V_{D} \cdot\left(\frac{1}{2 \pi}-\frac{1}{8} M \cos \phi\right) \\
& +\frac{f_{c} V_{R}}{2 S}\left(\frac{d I_{F}}{d t}\right)\left(\frac{S t_{r r}}{S+1}\right)^{2}
\end{aligned}
$$

\section{RESULTS}

An HEV traction drive was simulated over the FUDS cycle using Advanced VehIcle SimulatOR (ADVISOR), which is a user-friendly conventional, electric or hybrid vehicle simulator package programmed in MATLAB/SIMULINK environment by the U.S. Department of Energy Hybrid Program at the National Renewable Energy Laboratory. As a result of simulation, motor torque and speed profiles sampled at $1 \mathrm{~Hz}$ were obtained. From these profiles, current peak, $I$, and modulation index, $M$, profiles were calculated assuming $\mathrm{V} / \mathrm{Hz}$ control and following the following algorithm:

Algorithm to find $I$ and $M$

1. get $T_{e}$ and $\omega_{e}$ profiles from ADVISOR

2. Machine input power, $P_{i n}=\frac{T_{e} \cdot \omega_{r}}{\eta}$

3. Output frequency, $f_{o}=\frac{p}{2} \cdot \frac{\omega_{r}}{2 \pi}$

4. V/Hz constant, $K_{v}=\frac{\sqrt{\frac{3}{2}} \frac{4}{\pi} V_{d c}}{f_{b}}$, where $f_{b}$ is the base frequency

5. Rms line voltage $V_{L}=f_{o} \cdot K_{v}$

6. RMS line current, $I_{L}=\frac{P_{i n}}{\sqrt{3} V_{L} \cos \phi}$

7. Peak line current, $I=\sqrt{2} I_{L}$

8. Modulation index, $M=\frac{V_{L}}{\sqrt{\frac{3}{2}} \frac{4}{\pi} V_{d c}}$

Using $I$ and $M$ values, the device power losses are calculated. Fig. 7a shows a comparison of $\mathrm{Si}$ and $\mathrm{SiC}$ diode losses. $\mathrm{SiC}$ diodes do not have much of a reverse recovery current; therefore, their switching losses are low. The conduction losses are also low because of $\mathrm{SiC}$ properties. This is why SiC diode total losses are lower compared with those of the Si diode losses in the inverter. Fig. 7b, on the other hand, shows the total MOSFET losses. Although the switching losses of $\mathrm{Si}$ and $\mathrm{SiC}$ MOSFETs are similar, the big difference between their total losses is due to the conduction losses. The specific on-resistance for the $\mathrm{SiC}$ MOSFET is $0.3 \times 10^{-3} \Omega-\mathrm{cm}^{2}$; and for the Si MOSFET, it is $180 \times 10^{-3} \Omega-\mathrm{cm}^{2}$.

Fig. 8a shows the total device losses of the three-phase inverter. As seen from the figure, the Si inverter has high losses compared with that of the $\mathrm{SiC}$ inverter. Corresponding energy loss in the $\mathrm{Si}$ inverter is $902.9 \mathrm{~W} \cdot \mathrm{sec}$ and in the $\mathrm{SiC}$ inverter is $287.6 \mathrm{~W} \cdot \mathrm{sec}$ over the FUDS cycle. With lower device losses, the $\mathrm{SiC}$ inverter is expected to have a higher efficiency. Figs. $8 \mathrm{~b}$ and $8 \mathrm{c}$ show the motoring efficiency of the inverter. It is around $95 \%$ for the $\mathrm{SiC}$ inverter, while it fluctuates around $85-90 \%$ for the $\mathrm{Si}$ inverter. (Note that the zero efficiency points correspond to the instants where the motor is stopped or generating and there is no positive power flow through the inverter.). Higher efficiency also results in less need for recharging the battery.

The junction temperature profiles of the MOSFETs are calculated by feeding the loss profiles to the device thermal equivalent circuit in Fig. 9. For this example, the junction temperature profiles can be seen in Fig. 10. The heatsinks for the MOSFETs are chosen to limit the junction temperature to the rated values: $150^{\circ} \mathrm{C}$ for $\mathrm{Si}$ and $175^{\circ} \mathrm{C}$ (Infineon datasheet) for SiC. Theoretically, SiC devices can withstand higher temperatures. Therefore, another heatsink is selected from [8] to limit the $\mathrm{SiC}$ junction temperature to $325^{\circ} \mathrm{C}$. Calculations show that the $\mathrm{Si}$ MOSFET needs a large heatsink to prevent thermal damage, while the SiC MOSFET needs only a small one for either junction temperature limit. The same reasoning is 

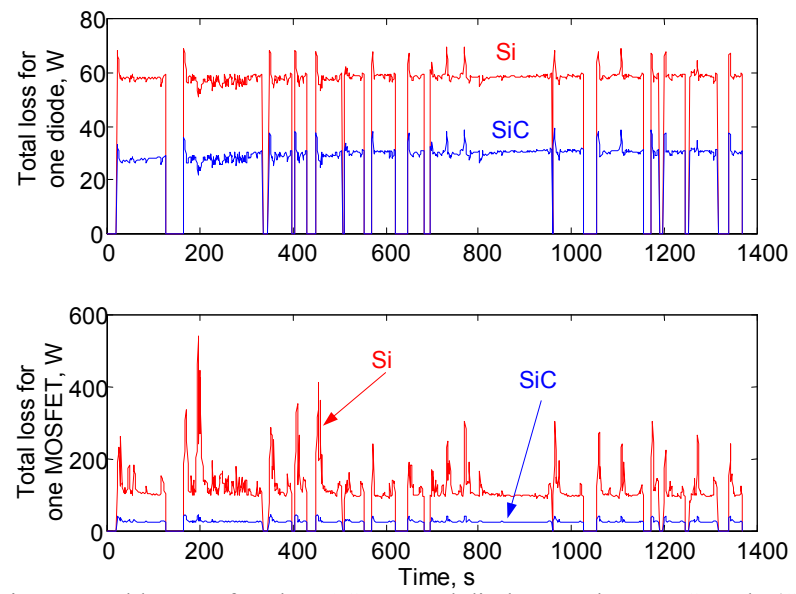

Fig. 7: Total losses of each MOSFET and diode over the FUDS cycle (Sired, top, and $4 \mathrm{H}-\mathrm{SiC}-\mathrm{blue})$

also valid for the diode junction temperature profiles, which are not shown here.

Normally, for the kind of inverter in this paper, watercooled heatsinks are used. However, for the ease of calculation, natural air-cooled heatsinks are considered here. For the whole inverter, six diodes and six MOSFETs should be taken into consideration. Calculations show that the amount of space saved just by using SiC MOSFETs instead of their $\mathrm{Si}$ counterparts is around $7500 \mathrm{~cm}^{3}$. The weight savings corresponding to this volume is $20.25 \mathrm{~kg}$. Note that, for this calculation, SiC MOSFET junction temperature is considered to be $175^{\circ} \mathrm{C}$. For the $325^{\circ} \mathrm{C}$ case, the savings are more. In an $\mathrm{HEV}$, size is extremely important because the amount of space available is limited. The weight reduction and efficiency increase result in an increase in the fuel economy of the vehicle.

\section{CONCLUSIONS}

In this paper, losses of an of a Si-based PWM inverter and a SiC-based PWM inverter are compared. Replacing Si-based power devices with SiC-based power devices brings many advantages for power conversion applications. The advantages of $\mathrm{SiC}$ based power electronics were discussed in previous sections.

The result of the loss studies showed an increase in efficiency with a decrease in the device losses when $\mathrm{SiC}$ is used. In addition, thermal studies showed that SiC-based converters need less cooling because of the material's superior thermal characteristics and because of lower
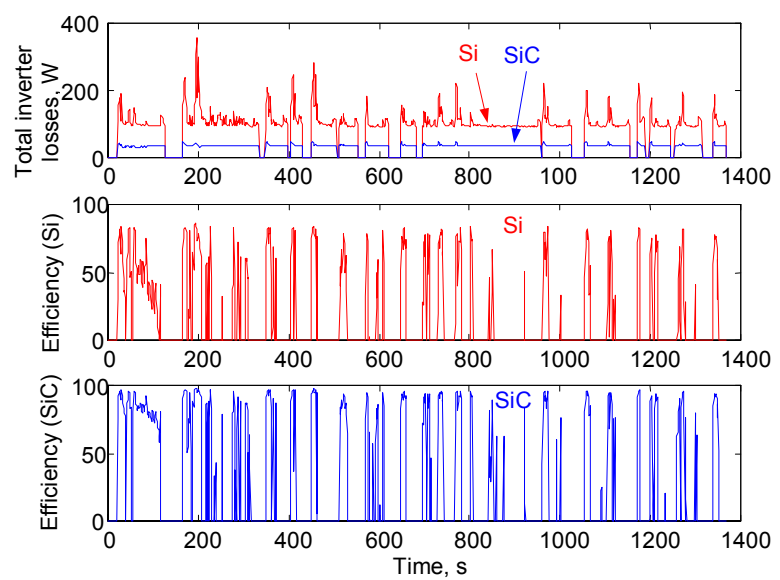

Fig. 8: Total losses and the efficiency of the inverter over the FUDS cycle (Si-red, top, and 4H-SiC-blue)

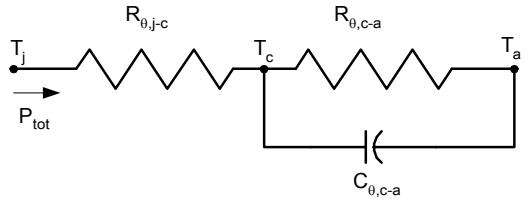

Fig. 9: Transient thermal model of a MOSFET

losses associated with $\mathrm{SiC}$ power devices.

When the processing issues are solved and the price of $\mathrm{SiC}$ wafers decreases, it is expected that $\mathrm{SiC}$ will replace $\mathrm{Si}$ in power devices, especially in medium to high-voltage range.

\section{REFERENCES}

[1] K. Shenai, R. S. Scott, B. J. Baliga, "Optimum semiconductors for high-power electronics," IEEE Transactions on Electron Devices, vol. 43, no: 9, pp. 1811-1823, Sept. 1989.

[2] A. Hefner, D. Berning, J. S. Lai, C. Liu, R. Singh, T. Kamgaing, J. Bernstein, "Silicon carbide merged PiN schottky diode switching characteristics and evaluation for power supply applications," IEEE IAS Annual Meeting Conf Proc., pp. 2948-2954, 2000.

[3] M. Bhatnagar and B. J. Baliga, "Comparison of 6H-SiC, 3C-SiC, and Si for power devices," IEEE Trans. on Electron Devices, 40 (3), pp. 645-655, March 1993.

[4] A. Elasses, M. Kheraluwala, M. Ghezzo, R. Steigerwald, N. Krishnamurthy, J. Kretchmer, and T. P. Chow, "A comparative evaluation of new silicon carbide diodes and state-of-the-art silicon diodes for power electronic applications," IEEE IAS Annual Meeting Conference Proceedings, pp. 341-345, 1999.

[5] K. Berringer, J. Marvin, P. Perruchoud, "Semiconductor power losses in ac inverters," IEEE IAS Annual Meeting Conf Proc., pp. 882-888, 1995.

[6] J. W. Kolar, H. Ertl, F. C. Zach, “ How to include the dependency of the $\mathrm{R}_{\mathrm{DS} \text { (on) }}$ of the power MOSFET's on the instantaneous value of the drain current into the calculation of the conduction losses of high-frequency three-phase PWM inverters," IEEE Transactions on Industrial Electronics, vol. 45, no. 3, June 1998.

[7] A. Q. Huang, B. Zhang, "Comparing SiC switching power devices: MOSFET, NPN transistor and GTO transistor," Solid State Electronics, Pergamon Press, pp. 325-340, 2000.

[8] N. Mohan, T. M. Undeland, W. P. Robbins, Power Electronics, 2nd Edition, John Wiley \& Sons Inc., 1995.

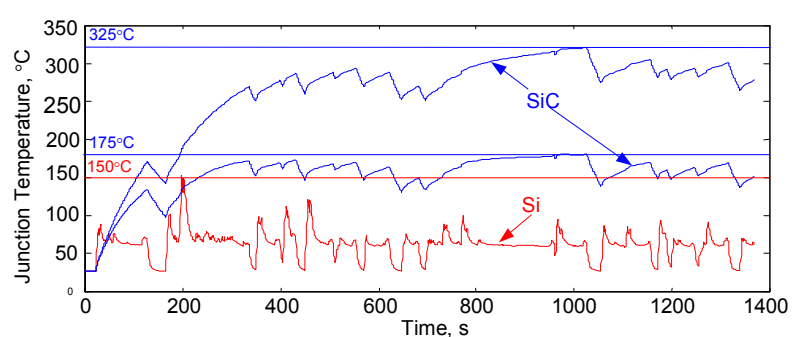

Fig. 10: Junction temperature profiles of a Si MOSFET and a $\mathrm{SiC}$ MOSFET in the three-phase inverter with heatsink (Si-red, bottom, and $4 \mathrm{H}-\mathrm{SiC}$-blue, top two) 